\title{
Microsurgical versus endovascular treatment of spinal epidural arteriovenous fistulas with intradural venous drainage: a multicenter study of 81 patients
}

\author{
Keisuke Takai, MD, PhD, ${ }^{8}$ Toshiki Endo, MD, PhD, ${ }^{2}$ Takao Yasuhara, MD, PhD, ${ }^{13}$ \\ Toshitaka Seki, MD, PhD, ${ }^{1}$ Kei Watanabe, MD, PhD, ${ }^{3}$ Yuki Tanaka, MD, ${ }^{3}$ Ryu Kurokawa, MD, PhD, ${ }^{4}$ \\ Hideaki Kanaya, MD, PhD, ${ }^{4}$ Fumiaki Honda, MD, PhD, ${ }^{5}$ Takashi Itabashi, MD, PhD, ${ }^{6}$ \\ Osamu Ishikawa, MD, PhD, ${ }^{7}$ Hidetoshi Murata, MD, PhD, ${ }^{9}$ Takahiro Tanaka, MD, ${ }^{9}$ \\ Yusuke Nishimura, MD, PhD, ${ }^{10}$ Kaoru Eguchi, MD, ${ }^{10}$ Toshihiro Takami, MD, PhD,11 \\ Yusuke Watanabe, MD, ${ }^{11}$ Takeo Nishida, MD, PhD, ${ }^{12}$ Masafumi Hiramatsu, MD, PhD, ${ }^{13}$ \\ Tatsuya Ohtonari, MD, PhD, ${ }^{14}$ Satoshi Yamaguchi, MD, PhD, ${ }^{15}$ Takafumi Mitsuhara, MD, PhD, ${ }^{15}$ \\ Seishi Matsui, MD, PhD, ${ }^{16}$ Hisaaki Uchikado, MD, PhD, ${ }^{17}$ Gohsuke Hattori, MD, PhD, ${ }^{17}$ \\ Nobutaka Horie, MD, PhD, ${ }^{18}$ Hitoshi Yamahata, MD, PhD, ${ }^{19}$ and Makoto Taniguchi, MD, PhD ${ }^{8}$ \\ 1Department of Neurosurgery, Hokkaido University Hospital, Sapporo; '2Department of Neurosurgery, Kohnan Hospital, Sendai; \\ ${ }^{3}$ Department of Orthopaedic Surgery, Niigata University Medical and Dental Hospital, Niigata; ${ }^{4}$ Department of Neurosurgery, \\ Dokkyo Medical University Hospital, Tochigi; ${ }^{5}$ Department of Neurosurgery, Gunma University Hospital, Gunma; ${ }^{6}$ Department \\ of Orthopaedic Surgery, Japanese Red Cross Narita Hospital, Chiba; ${ }^{7}$ Department of Neurosurgery, The University of Tokyo \\ Hospital, Tokyo; ${ }^{8}$ Department of Neurosurgery, Tokyo Metropolitan Neurological Hospital, Tokyo; ${ }^{9}$ Department of Neurosurgery, \\ Yokohama City University Hospital, Yokohama; ${ }^{10}$ Department of Neurosurgery, Nagoya University Hospital, Nagoya; \\ ${ }^{11}$ Department of Neurosurgery, Osaka City University Graduate School of Medicine, Osaka; ${ }^{12}$ Department of Neurosurgery, \\ Osaka University Graduate School of Medicine, Osaka; ${ }^{13}$ Department of Neurosurgery, Okayama University Graduate School \\ of Medicine, Okayama; ${ }^{14}$ Department of Spinal Surgery, Brain Attack Center, Ota Memorial Hospital, Hiroshima; ${ }^{15}$ Department \\ of Neurosurgery, Hiroshima University Hospital, Hiroshima; ${ }^{16}$ Department of Neurosurgery, Ehime University Hospital, Ehime; \\ ${ }^{17}$ Department of Neurosurgery, Kurume University Hospital, Fukuoka; ${ }^{18}$ Department of Neurosurgery, Nagasaki University \\ Hospital, Nagasaki; and ${ }^{19}$ Department of Neurosurgery, Kagoshima University Hospital, Kagoshima, Japan
}

OBJECTIVE Spinal arteriovenous shunts are rare vascular lesions and are classified into 4 types (types I-IV). Due to rapid advances in neuroimaging, spinal epidural AVFs (edAVFs), which are similar to type I spinal dural AVFs (dAVFs), have recently been increasingly reported. These 2 entities have several important differences that influence the treatment strategy selected. The purposes of the present study were to compare angiographic and clinical differences between edAVFs and dAVFs and to provide treatment strategies for edAVFs based on a multicenter cohort.

METHODS A total of 280 consecutive patients with thoracic and lumbosacral spinal dural arteriovenous fistulas (dAVFs) and edAVFs with intradural venous drainage were collected from 19 centers. After angiographic and clinical comparisons, the treatment failure rate by procedure, risk factors for treatment failure, and neurological outcomes were statistically analyzed in edAVF cases.

RESULTS Final diagnoses after an angiographic review included 199 dAVFs and 81 edAVFs. At individual centers, 29 patients (36\%) with edAVFs were misdiagnosed with dAVFs. Spinal edAVFs were commonly fed by multiple feeding arteries (54\%) shunted into a single or multiple intradural vein(s) (91\% and $9 \%$ ) through a dilated epidural venous plexus. Preoperative modified Rankin Scale (mRS) and Aminoff-Logue gait and micturition grades were worse in patients with edAVFs than in those with dAVFs. Among the microsurgical $(n=42)$, endovascular $(n=36)$, and combined $(n=3)$ treat-

ABBREVIATIONS AVM = arteriovenous malformation; dAVF = dural arteriovenous fistula; edAVF = epidural AVF; mRS = modified Rankin Scale; NBCA = N-butyl-cyanoacrylate. SUBMITTED November 30, 2019. ACCEPTED February 18, 2020.

INCLUDE WHEN CITING Published online April 24, 2020; DOI: 10.3171/2020.2.SPINE191432. 
ment groups of edAVFs, the treatment failure rate was significantly higher in the index endovascular treatment group (7.5\%,31\%, and $0 \%$, respectively). Endovascular treatment was found to be associated with significantly higher odds of initial treatment failure (OR 5.72, 95\% Cl 1.45-22.6). In edAVFs, the independent risk factor for treatment failure after microsurgery was the number of intradural draining veins (OR 17.9, 95\% Cl 1.56-207), while that for treatment failure after the endovascular treatment was the number of feeders (OR 4.11, 95\% CI 1.23-13.8). Postoperatively, mRS score and Aminoff-Logue gait and micturition grades significantly improved in edAVFs with a median follow-up of 31 months.

CONCLUSIONS Spinal epidural AVFs with intradural venous drainage are a distinct entity and may be classified as type $V$ spinal vascular malformations. Based on the largest multicenter cohort, this study showed that primary microsurgery was superior to endovascular treatment for initial treatment success in patients with spinal edAVFs.

https://thejns.org/doi/abs/10.3171/2020.2.SPINE191432

KEYWORDS spinal vascular malformations; spinal arteriovenous shunts; dural arteriovenous fistulas; extradural arteriovenous fistulas; surgery; endovascular procedures; vascular disorders

$\mathrm{S}$ PINAL arteriovenous (AV) shunts are rare vascular lesions characterized by abnormal direct connection(s) between spinal artery(ies) and vein(s) without an intervening blood capillary system. ${ }^{1}$ In the 1980 s and 1990 s, they were classified into 4 types according to the fistulous location and configuration: type I, dural arteriovenous fistulas (dAVFs); type II, intradural glomus arteriovenous malformations (AVMs); type III, intradural-extradural juvenile AVMs; and type IV, intradural perimedullary AVFs. ${ }^{1,2}$ Due to rapid advances in neuroimaging, spinal epidural AVFs (edAVFs) that cannot be classified into any type have been increasingly reported recently; ${ }^{3-5}$ edAVFs were frequently misdiagnosed as dAVFs because their angiographic and clinical characteristics mimic dAVFs; furthermore, no standard treatment has been established for edAVFs. ${ }^{6,7}$ The present study was conducted to 1) clarify key angiographic and clinical characteristics of patients with spinal edAVFs, and 2) provide treatment strategies for these patients based on a large multicenter cohort.

\section{Methods \\ Ethics}

This study protocol was approved by the institutional review board at the Tokyo Metropolitan Neurological Hospital and participating centers. Since this was a retrospective and noninvasive study, written informed consent from patients was waived. A public notice that provided information on this study was instead given on each center's website.

\section{Patient Population}

Consecutive patients with thoracic and lumbosacral dAVFs and edAVFs with intradural venous drainage who were treated microsurgically and/or endovascularly between 2009 and 2018 were included. Patients with an at least 6-month follow-up after the index treatment were included. Patients with cervical AV shunts, intradural AV shunts, edAVFs without intradural venous drainage, no angiographic data, and no long-term follow-up clinical data ( $<6$ months) were excluded. Angiographic and clinical data were collected using a standardized form from 19 centers in Japan (Fig. 1). Participating centers were selected from among centers certified by the Japan Neurosurgical Society, Neurospinal Society of Japan, or Japanese Society for Spine Surgery and Related Research based on an established reputation in the treatment of spinal AV shunts and an expressed interest in study participation. Among the 19 centers, the first choice of treatment is microsurgery in 9 centers and endovascular treatment in 4 centers; in 6 centers, the decision to perform surgery or embolization is made through an expert consensus conference.

\section{Angiographic Review}

Spinal edAVFs with intradural venous drainage were defined as spinal AVFs that develop in the spinal epidural space, are fed by branches of the segmental artery, and shunt into the intradural vein through a dilated epidural venous plexus. Preoperative and postoperative angiograms were reviewed by the neuroradiologists in each center, who were blinded to the purpose of the present study. Angiographic data were rereviewed, and final diagnoses were made by 3 neurosurgeons (K.T., T.E., and T.Y.) certified by the Neurospinal Society of Japan. Reviewers had more than 20 years of experience in spine surgery. Differential diagnoses of edAVFs from dAVFs at the individual hospitals were compared with final diagnoses.

\section{Data Analysis}

The outcomes of interest included 1) differences in angiographic and clinical characteristics between dAVFs and edAVFs, 2) the rate of treatment failure by procedure, 3) risk factors for treatment failure, 4) specific treatment strategies, and 5) neurological outcomes in edAVFs. The following angiographic data were compared between dAVFs and edAVFs: the number of feeding arteries, location of the fistula, and number of intradural draining veins. The following clinical data were compared between patients with dAVFs and edAVFs: age, sex, symptoms, the mean duration of symptoms, preoperative modified Rankin Scale (mRS) score, Aminoff-Logue gait grade, AminoffLogue micturition grade, the presence of pain/numbness, and a previous history of spine surgery or trauma.

In edAVFs, the following variables regarding treatment outcomes were compared among the microsurgical, endovascular, and combined treatment groups: operators' experience, the treatment failure rate, and major complications. Major complications included cerebrospinal fluid leakage, surgical site infection, postoperative hematoma, spinal cord infarction, catheter perforation, deep vein thrombosis, and pulmonary embolism. The combined treatment 


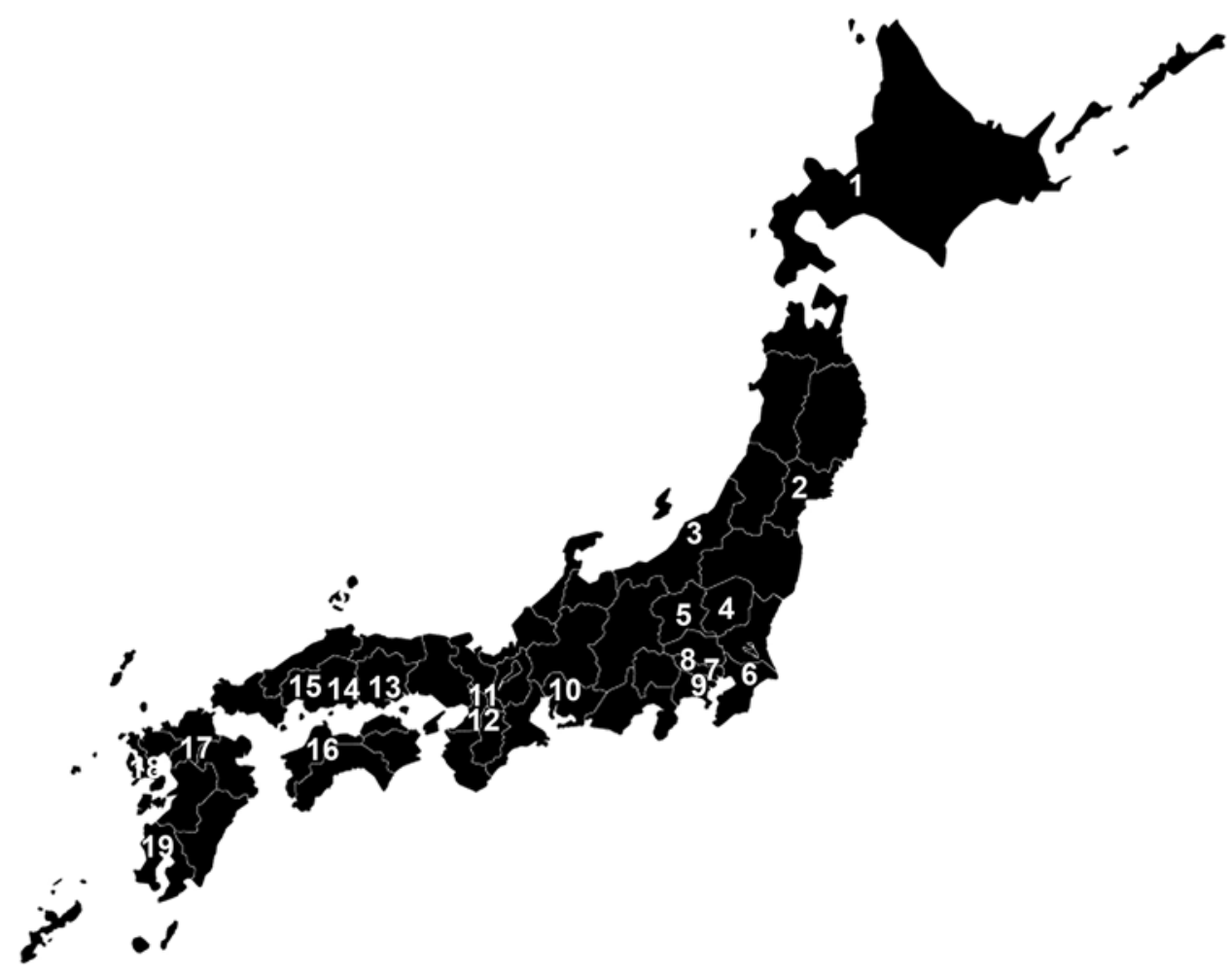

FIG. 1. Geographic distribution of the 19 centers in Japan and the distribution of patients with edAVFs by center: 1, Department of Neurosurgery, Hokkaido University Hospital $(n=5) ; 2$, Department of Neurosurgery, Kohnan Hospital $(n=7) ; 3$, Department of Orthopedic Surgery, Niigata University Medical and Dental Hospital $(n=6) ; 4$, Department of Neurosurgery, Dokkyo Medical University Hospital $(n=4) ; 5$, Department of Neurosurgery, Gunma University Hospital $(n=3) ; 6$, Department of Orthopedic Surgery, Japanese Red Cross Narita Hospital $(n=6) ; 7$, Department of Neurosurgery, The University of Tokyo Hospital $(n=2)$; 8, Department of Neurosurgery, Tokyo Metropolitan Neurological Hospital $(n=7) ; 9$, Department of Neurosurgery, Yokohama City University Hospital $(n=3) ; 10$, Department of Neurosurgery, Nagoya University Hospital $(n=8) ; 11$, Department of Neurosurgery, Osaka City University Hospital $(n=4) ; 12$, Department of Neurosurgery, Osaka University Hospital $(n=5)$; 13, Department of Neurosurgery, Okayama University Hospital $(n=8) ; 14$, Department of Spinal Surgery, Brain Attack Center, Ota Memorial Hospital $(n=1) ; 15$, Department of Neurosurgery, Hiroshima University Hospital $(n=2) ; 16$, Department of Neurosurgery, Ehime University Hospital $(n=3) ; 17$, Department of Neurosurgery, Kurume University Hospital $(n=1) ; 18$, Department of Neurosurgery, Nagasaki University Hospital $(n=3) ; 19$, Department of Neurosurgery, Kagoshima University Hospital $(n=3)$. The map was downloaded from http://www.start-point.net.

was defined as a planned microsurgical and endovascular treatment. A patient with a fistula that required additional microsurgery or endovascular treatment for obliteration after the index treatment was defined as a case of treatment failure. Treatment failure included initial treatment failure and late recurrence. Patients for whom more than 1 treatment was performed were regarded as a single case; only the index treatment was registered.

In edAVFs, the following variables regarding neurological outcomes were compared between the treatment failure and success groups: postoperative mRS score, Aminoff-Logue gait grade, Aminoff-Logue micturition grade, and changes in pain/numbness. The AminoffLogue grades for gait (range $0-5$ ) and micturition (range $0-3$ ) are as follows: ${ }^{8}$ gait grade 0 , normal; 1 , leg weakness, abnormal gait or stance, but no restriction of activity; 2 , restricted activity but not requiring support; 3 , requiring 1 stick for walking; 4 , requiring 2 sticks, crutches, or walker; and 5, confined to wheelchair; micturition grade 0 , normal; 1 , hesitancy, urgency, or frequency; 2 , occasional urinary incontinence or retention; and 3, total incontinence or retention. The neurological outcomes of patients were reviewed from medical charts or telephone interviews by the clinicians in each center who were blinded to the purpose of the present study between February and June 2019.

\section{Statistical Analysis}

Nominal variables are expressed as counts and percentages, and continuous variables that follow a normal distribution are expressed as means with standard deviations. Continuous variables that did not follow a normal distribution are expressed as medians and ranges. Ordinal variables are expressed as medians and interquartile ranges. Fisher's exact test or the chi-square test for nominal variables; the Student t-test for continuous variables; and the Mann-Whitney U-test, the Kruskal-Wallis test, or Wilcoxon signed-rank test for continuous variables that did not follow normal distributions or ordinal variables were used to statistically analyze the data collected. The survival times for additional treatment after the index treatment was calculated using the Kaplan-Meier method and were compared among treatment procedures with the log-rank 
TABLE 1. Angiographic and clinical characteristics of edAVFs and dAVFs

\begin{tabular}{|c|c|c|c|}
\hline Variable & $\begin{array}{l}\text { edAVF } \\
(n=81)\end{array}$ & $\begin{array}{c}\text { dAVF } \\
(n=199)\end{array}$ & $\begin{array}{c}p \\
\text { Value }\end{array}$ \\
\hline \multicolumn{4}{|l|}{ Feeder } \\
\hline Median no. (range) & $2(1-6)$ & $1(1-3)$ & $<0.001^{*}$ \\
\hline Multiple levels \&/or bilateral, n (\%) & $44(54)$ & $27(14)$ & $<0.001^{*}$ \\
\hline \multicolumn{4}{|l|}{ Fistula, $\mathrm{n}(\%)$} \\
\hline Single level & $81(100)$ & $195(98)$ & 0.327 \\
\hline Lumbosacral level & $67(83)$ & $37(19)$ & $<0.001^{*}$ \\
\hline Ventral & $76(94)$ & $6(3)$ & $<0.001^{*}$ \\
\hline Lt side & $53(65)$ & $107(54)$ & 0.184 \\
\hline \multicolumn{4}{|l|}{ Intradural drainer } \\
\hline Median no. (range) & $1(1-5)$ & $1(1-2)$ & $<0.001^{*}$ \\
\hline Multiple levels \&/or bilateral, n (\%) & $7(9)$ & $0(0)$ & $<0.001^{*}$ \\
\hline \multicolumn{4}{|l|}{ Age \& sex } \\
\hline Mean age $\pm S D$, yrs & $67 \pm 11$ & $66 \pm 11$ & 0.552 \\
\hline Male sex, $\mathrm{n}(\%)$ & $59(73)$ & $165(83)$ & 0.070 \\
\hline \multicolumn{4}{|l|}{ Presentation } \\
\hline $\begin{array}{l}\text { Mean duration of symptoms } \pm \text { SD, } \\
\text { mos }\end{array}$ & $16 \pm 24$ & $20 \pm 36$ & 0.150 \\
\hline Median mRS score (IQR) & $4(3-4)$ & $3(2-4)$ & $<0.001^{*}$ \\
\hline $\begin{array}{l}\text { Median Aminoff-Logue gait grade } \\
\quad(\mathrm{IQR})\end{array}$ & $4(3-5)$ & $3(2-4)$ & $<0.001^{*}$ \\
\hline $\begin{array}{l}\text { Median Aminoff-Logue micturition } \\
\text { grade (IQR) }\end{array}$ & $2(1-3)$ & $2(1-2)$ & $<0.001^{*}$ \\
\hline Pain/numbness, n (\%) & $77(95)$ & $184(92)$ & 0.602 \\
\hline \multicolumn{4}{|l|}{ Previous treatment or trauma, $\mathrm{n}(\%)$} \\
\hline Spine surgery or fracture & $11(14)$ & $17(8.5)$ & 0.271 \\
\hline $\begin{array}{l}\text { Spine surgery or fracture adjacent } \\
\text { to the fistula }\end{array}$ & $11(14)$ & $3(1.5)$ & $<0.001^{*}$ \\
\hline
\end{tabular}

* Significant difference.

test. Odds ratios and 95\% confidence intervals for initial treatment failure after endovascular treatment were calculated using the Mantel-Haenszel chi-square test. Univariate logistic regression analyses were performed to identify angiographic risk factors for treatment failure after microsurgical or endovascular treatment. IBM SPSS Statistics (version 23, IBM Corp.) software was used to conduct the analysis. A 2-tailed $\mathrm{p}<0.05$ was considered to be significant.

\section{Results \\ Patients}

A total of 318 patients were enrolled in this study, and 38 were subsequently excluded because of cervical $\mathrm{AV}$ shunts (5 patients), intradural AV shunts (6 patients), edAVFs without intradural venous drainage (5 patients), no angiographic data (2 patients), no long-term follow-up clinical data (10 patients), and treatment before 2009 (10 patients). Therefore, 280 patients were included in this study.

\section{Initial and Final Diagnoses}

The initial diagnoses of AVFs at the 19 individual centers were 227 spinal dAVFs and 53 spinal edAVFs. Final diagnoses after reviews of angiographic data were 199 spinal dAVFs and 81 spinal edAVFs. Twenty-nine cases (36\%) of edAVFs were misdiagnosed as dAVFs at individual centers, whereas 1 case of dAVF was misdiagnosed as edAVF. The cause of misdiagnoses was mainly edAVFs being under-recognized; thus, specific neuroimaging findings were overlooked.

\section{Angiographic Characteristics}

Several dissimilarities in the angiographic findings of the 2 groups were evident (Table 1). Spinal dAVFs were commonly fed by a single feeder (86\%), developed in the dorsal dura mater in the thoracic region, and shunted into a single intradural vein through the dura mater. In dAVFs, the fistulous connection between feeder(s) and the intradural vein was located at a single spinal level in 195 patients (98\%) and at 2 independent spinal levels in 4 (2\%). In contrast, spinal edAVFs were commonly fed by multiple (multilevel and/or bilateral) feeders (54\%), developed in the ventral epidural space in the lumbosacral region, and shunted into a single or multiple intradural vein(s) $(91 \%$ and $9 \%$, respectively) through a dilated epidural venous plexus (Fig. 2). In edAVFs, the fistulous connection between feeder(s) and the dilated epidural venous plexus was located at a single spinal level in all 81 cases (100\%). In edAVFs, the ventral epidural venous plexus was located at a single vertebral level in 66 patients $(81 \%)$ and multiple vertebral levels in 15 (19\%). In edAVFs, multiple intradural venous drainers were more commonly observed in patients with a large ( 2 or more spinal levels) venous plexus (Fig. 3, p < 0.001). In edAVFs, venous drainage was observed in the intradural vein only in 63 patients (78\%) but was observed in both the intradural and paravertebral veins in 18 patients $(22 \%)$.

\section{Clinical Characteristics}

The clinical characteristics of patients with dAVFs and edAVFs were very similar for age, sex, symptoms, and the mean duration of symptoms; dAVFs and edAVFs both developed more frequently in older males in their $60 \mathrm{~s}$, and most patients presented with venous congestive myelopathy; the mean duration of symptoms before treatment was long (16-20 months). A high T2 signal in the spinal cord and/or flow voids around the spinal cord were observed in most patients with dAVFs (93\%) and edAVFs (99\%). However, preoperative symptoms were more severe in patients with edAVFs than in those with dAVFs as determined by mRS scores and Aminoff-Logue gait and micturition grades $(\mathrm{p}<0.001$, Table 1$)$.

\section{Previous History of Spine Surgery or Trauma}

The most common prior treatment or trauma associated with dAVFs and edAVFs was spinal laminectomy, fusion, or fracture. In most patients with dAVFs, the spinal levels of laminectomy, fusion, or fracture were away from the fistula level; however, in all patients with edAVFs, they were adjacent to the fistula level $(\mathrm{p}<0.001$, Table 1$)$. 
Takai et al.

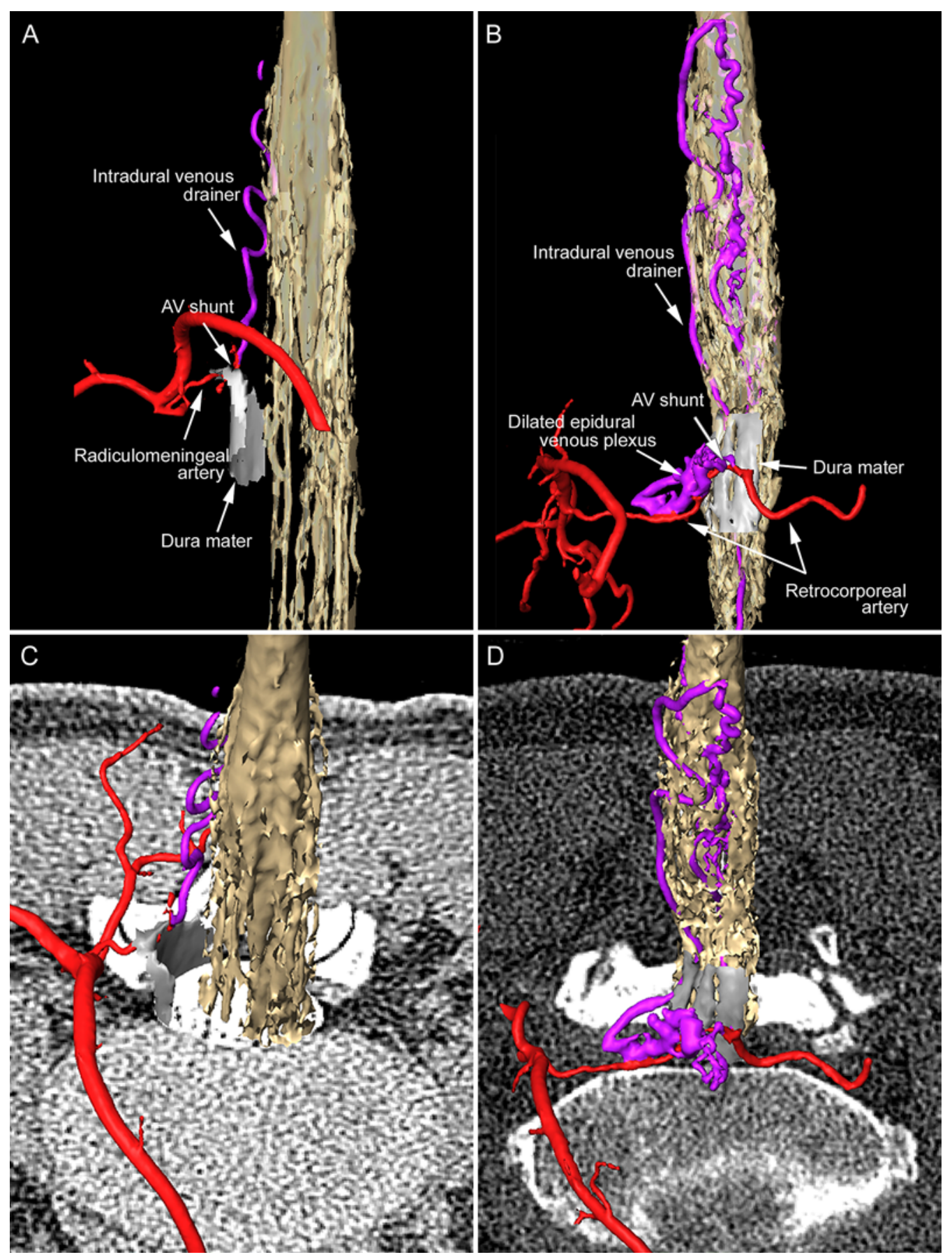

FIG. 2. Three-dimensional computer graphics image showing the angioarchitecture of spinal dAVFs (A and C) and edAVFs (B and D). Spinal dAVFs are fed by a radiculomeningeal artery, developed in the dorsal dura mater, and shunt into a single intradural vein through the dura mater. In contrast, spinal edAVFs are fed by bilateral retrocorporeal arteries, developed in the ventral epidural space, and shunt into a single or multiple intradural vein(s) through a dilated epidural venous plexus. Figure is available in color online only.

\section{Treatment Failure in edAVFs}

Among the microsurgical $(n=42)$, endovascular $(n=$ $36)$, and combined $(n=3)$ treatment groups of edAVFs, the rate of treatment failure was significantly higher in the index endovascular treatment group (microsurgical group, $7.5 \%$; endovascular group, $31 \%$; and combined group, $0 \%$; $\mathrm{p}=0.018$ [Fig. 4]), while operators' experience did not significantly differ among the 3 groups (microsurgical group, median 20 years [range 9-37 years]; endovascular group, 18 years [range 9-29 years]; and combined group, 18 years [range 17-23 years]; $p=0.799$ ). Endovascular treatment was found to be associated with significantly higher odds 
Takai et al.
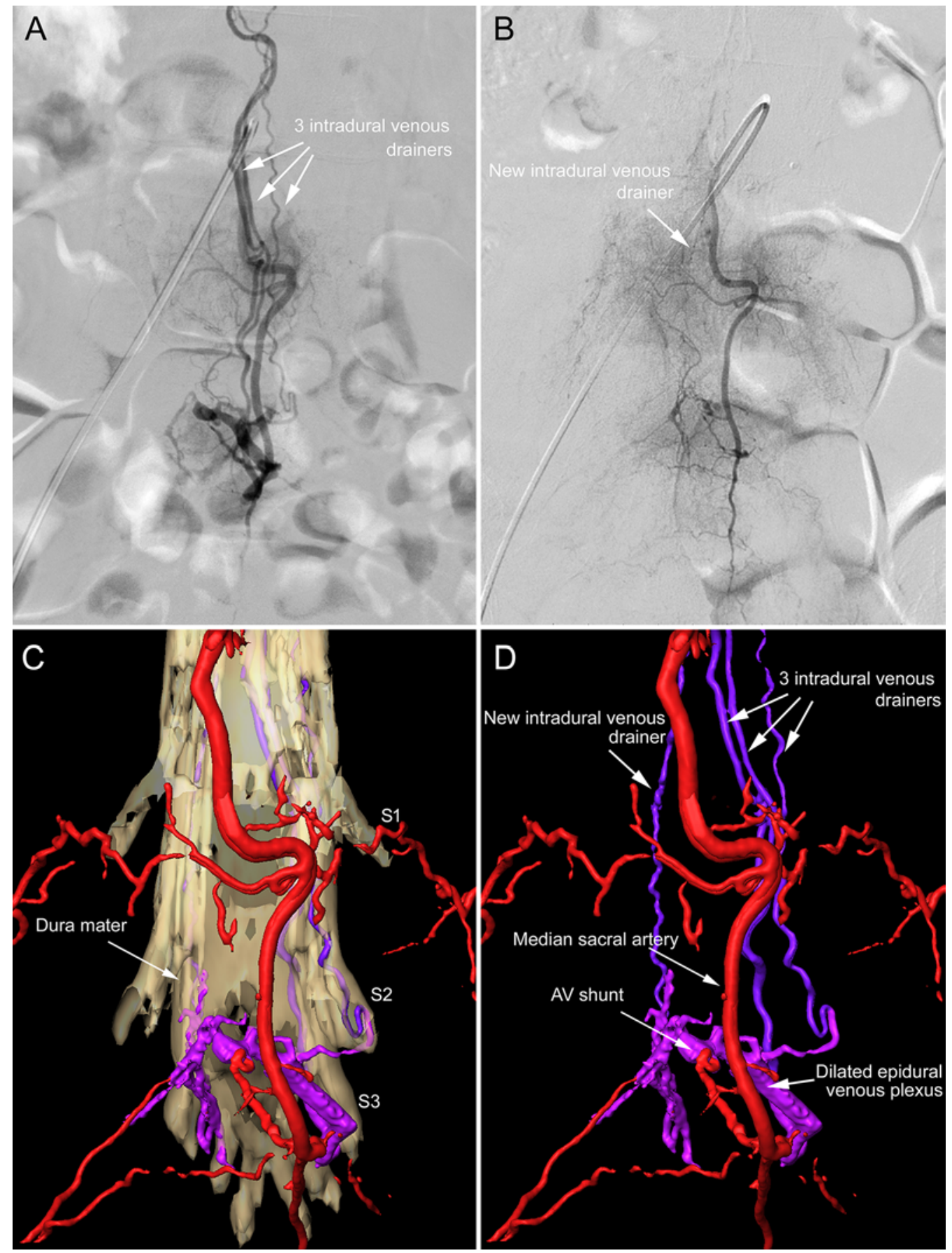

FIG. 3. Images obtained in a 61-year-old female patient with an edAVF. A: The patient was diagnosed with an edAVF fed by the median sacral artery that drained into 3 intradural veins as seen on the angiogram. She underwent microsurgical interruption of the 3 intradural venous drainers. B-D: However, postoperative angiography (B) showed a new intradural drainer that had not been recognized before surgery on the contralateral side, as shown in the 3D computer graphics images $(C$ and $D)$. She underwent additional microsurgical interruption of this vein, which led to the complete obliteration of the AVF. Her Aminoff-Logue gait grade improved after microsurgery. No recurrence was noted in the 10-month follow-up period. Figure is available in color online only.

of initial treatment failure (OR 5.72, 95\% CI 1.45-22.6; $\mathrm{p}$ $=0.013)$ than microsurgery. The imaging modalities used to detect treatment failure differed among each center. Among the 81 patients with edAVFs, 47 (58\%) were offered postoperative angiography $(\mathrm{n}=17)$ or $\mathrm{CT}$ angiography $(\mathrm{n}=$ 30), while all patients, except for one who had a history of cardiac pacemaker placement, were offered postoperative MRI and clinical evaluations.

\section{Risk Factors for Treatment Failure in edAVFs}

After univariate analyses, the independent risk factor for treatment failure after microsurgery was the number 


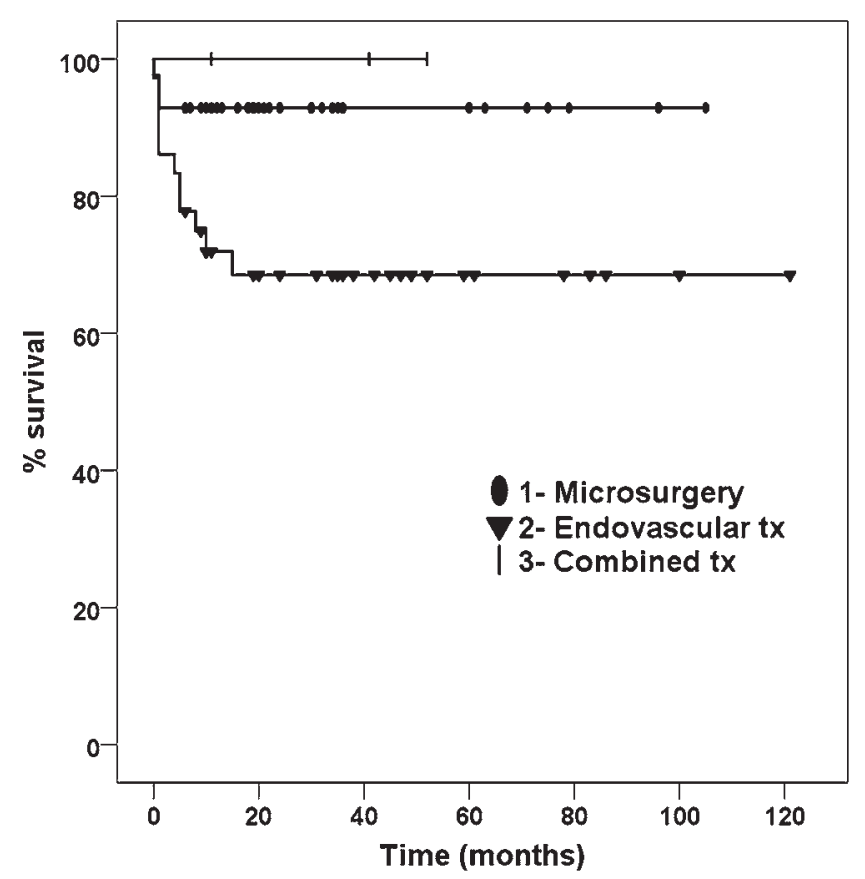

FIG. 4. Percent survival analysis of additional treatment (tx) after the index treatment by procedure. A Kaplan-Meier analysis shows longer progression-free survival with index microsurgery and the combined treatment than with the endovascular treatment $(p=0.022)$.

of intradural drainers (OR 17.9, 95\% CI 1.56-207; p = 0.021 , while that for treatment failure after endovascular treatment was the number of feeders (OR 4.11, 95\% CI 1.23-13.8; $\mathrm{p}=0.022)($ Table 2).

\section{Microsurgical Treatment of edAVFs}

In 42 patients with edAVFs treated by microsurgery, 34 (81\%) underwent microsurgical interruption of the proximal part of the intradural drainer alone, and 8 underwent coagulation of the dilated epidural venous plexus as well as the intradural drainer. The mean number of laminae removed during laminectomy or laminotomy was $1.9 \pm 0.6$ $( \pm$ SD) per procedure. Hemilaminectomy was performed in $28(67 \%)$ of the 42 patients. Intraoperative indocyanine green angiography was performed in 31 (74\%) of 42 patients.

In the microsurgical group, 3 patients $(7.5 \%)$ required an additional treatment after the index surgery because of treatment failure. Treatment failure was identified on early postoperative angiography within 1 month of index surgery without neurological decline. All 3 AVFs had multiple intradural venous drainers (median 2 drainers, range 2-4 drainers). Two of the 3 patients underwent interruption of a single intradural drainer alone as the index treatment; however, postoperative angiography revealed another new vein that had not been recognized before the index surgery as well as a persistent epidural venous plexus. The other patient underwent interruption of 3 intradural drainers and the dilated epidural venous plexus; however, postoperative angiography revealed another new vein that had not been recognized before the index surgery as well as a persistent epidural venous plexus (Fig. 3). All 3 patients underwent
TABLE 2. Angiographic risk factors associated with treatment failure in patients with edAVFs

\begin{tabular}{lcc}
\hline \multicolumn{1}{c}{ Risk Factor } & OR $(95 \%$ Cl $)$ & p Value \\
\hline Microsurgery & & \\
\hline No. of feeders & $0.54(0.07-4.34)$ & 0.563 \\
\hline Thoracic level of the fistula & $1.67(0.14-20.6)$ & 0.691 \\
\hline Multilevel epidural venous plexus & $4.11(0.99-17.0)$ & 0.051 \\
\hline No. of intradural drainers & $17.9(1.56-207)$ & $0.021^{*}$ \\
\hline Endovascular treatment & & \\
\hline No. of feeders & $4.11(1.23-13.8)$ & $0.022^{*}$ \\
\hline Thoracic level of the fistula & $9.00(0.82-99.3)$ & 0.073 \\
\hline Multilevel epidural venous plexus & $0.94(0.26-3.39)$ & 0.930 \\
\hline No. of intradural drainers & $0.84(0.36-1.98)$ & 0.695 \\
\hline Concentration of NBCA & $0.98(0.89-1.08)$ & 0.727 \\
\hline * Signiflan
\end{tabular}

* Significant difference.

additional microsurgical interruption of the intradural drainer, which led to complete obliteration of the AVF. No surgical complications occurred in patients treated with microsurgery.

Of the 34 patients who underwent microsurgical interruption of the intradural drainer alone, 16 were offered postoperative angiography or 3D CT angiography. In 12 (75\%) of these patients, the epidural venous plexus spontaneously occluded, while the venous plexus was left intact during surgery (Fig. 5). In the remaining 4 patients, the epidural venous plexus persisted; however, intradural venous drainage did not recur in the follow-up period.

Of the 42 patients with edAVFs treated by microsurgery, all, except for one with a history of cardiac pacemaker placement, were offered postoperative MRI at a median follow-up of 23 months (range 6-105 months). The high T2 signal in the spinal cord and flow voids decreased or disappeared in $40(98 \%)$ of the 41 patients with edAVFs.

\section{Endovascular Treatment of edAVFs}

In 36 patients with edAVFs treated by endovascular embolization, 33 underwent transarterial embolization with $\mathrm{N}$-butyl-cyanoacrylate (NBCA), 2 underwent transarterial embolization with coils, and 1 had transvenous embolization with NBCA. None of the endovascularly treated patients were treated using Onyx embolic material because it was not approved for patients with spinal AV shunts by the Pharmaceuticals and Medical Devices Agency in Japan. Of the 36 procedures, 16 resulted in occlusion of the feeder only; 10 in occlusion of the feeder and the dilated epidural venous plexus; and 9 in occlusion of the feeder, the dilated epidural venous plexus, and the proximal portion of the intradural drainer (Fig. 6). Treatment information was not available in the remaining patient. Among the 36 patients, 24 underwent embolization under local anesthesia and 12 under general anesthesia. No patients were contraindicated against general anesthesia because of medical comorbidities.

In the endovascular group, 11 patients $(31 \%)$ required an additional treatment because of treatment failure; 10 originally underwent transarterial embolization with 


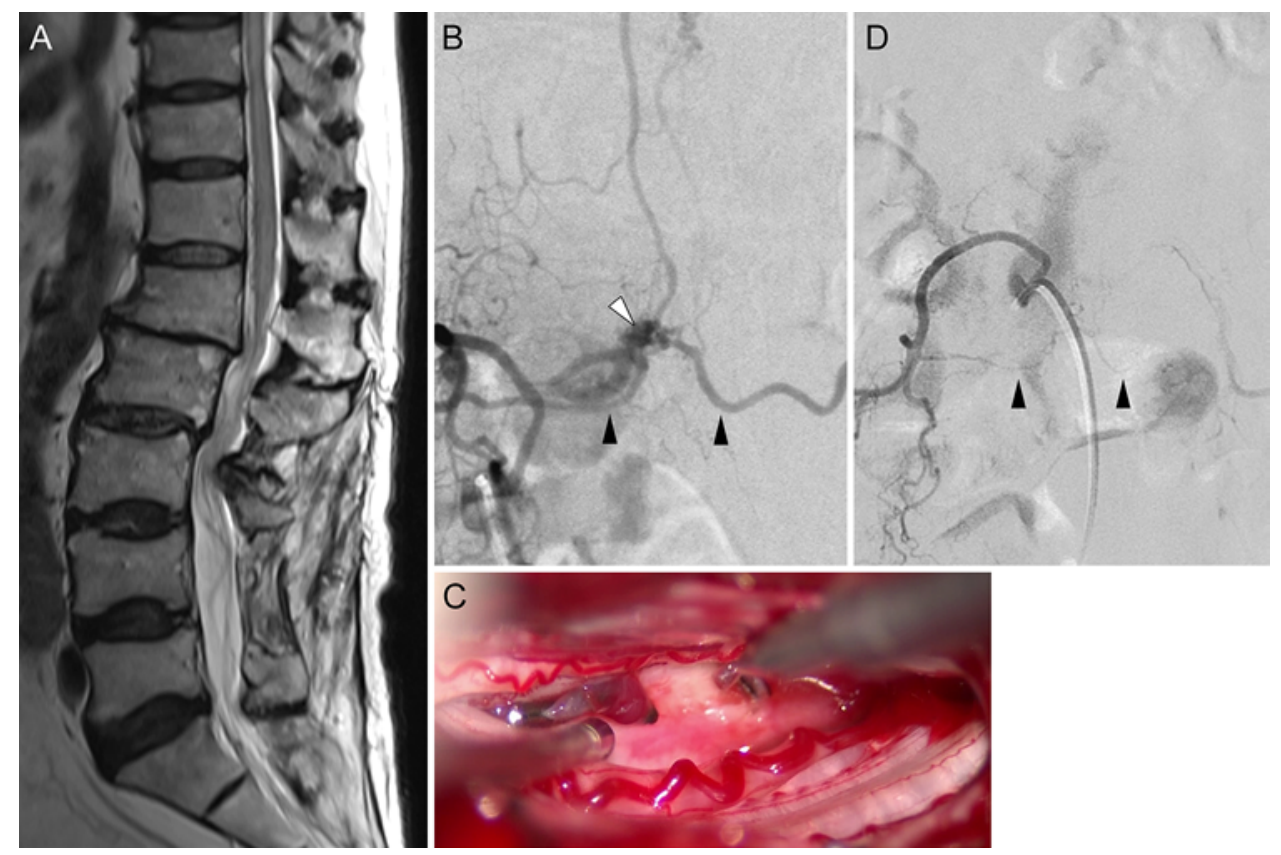

FIG. 5. Images obtained in a 68-year-old male patient with an edAVF. The patient had a previous history of intermittent claudication due to lumbar spinal stenosis that was treated with lumbar laminectomy and fusion at the L3 -5 levels at another hospital. Six years later, he presented with gait difficulty and incontinence. The pedicle screws and rods were removed, and a laminectomy at L2 was performed at the same hospital; however, his neurological deficits did not improve. A: Spinal sagittal MR image showing a high T2 signal in the lower spinal cord. B: Angiogram showing the edAVF (white arrowhead) fed by the bilateral L2 segmental arteries (black arrowheads) at the L2 level adjacent to the spinal fusion. The development of the edAVF may have been associated with lumbar fusion surgery. C: The patient underwent microsurgical interruption of a single intradural venous drainer, which led to the complete obliteration of the AVF. D: Note that the dilated epidural venous plexus spontaneously occluded but remained intact during microsurgery. The black arrowheads indicate the bilateral feeding arteries. After microsurgery, the patient's Aminoff-Logue gait and micturition grades improved. No recurrence was noted in the 35 -month follow-up period. Figure is available in color online only.

NBCA and the other underwent transarterial embolization with coils as an index treatment. Among the 11 patients, treatment failure was identified on early postoperative angiography within 1 month of index treatment without neurological decline in 7; however, it was associated with neurological decline in 4 . Treatment failure was identified between 5 and 15 months after the initial treatment in these 4 patients.

Of the 11 edAVFs, 8 were fed by more than 1 feeder (median of 3 feeders, range 2-6). In all 11 patients with treatment failure, the index treatment resulted in feeder occlusion only. In contrast, in all 19 patients in whom the index treatment resulted in occlusion of the epidural venous plexus, complete obliteration of the AVF was achieved, regardless of whether the intradural drainer was occluded (Fig. 6). The concentration of NBCA was not associated with treatment failure (initial success group, $24.3 \%$; treatment failure group, $23.3 \%$ ). Of the 11 patients with treatment failure, 6 underwent additional microsurgery and 5 underwent endovascular embolization. After the second treatment, complete obliteration of the AVF was achieved in all 11 patients. Regarding complications, retroperitoneal hemorrhage occurred in 1 patient treated endovascularly and was cured by conservative management.

Of the 36 patients with edAVFs treated by endovascular embolization, all, except for one with a history of cardiac pacemaker placement, were offered postoperative MRI in the median follow-up of 40 months (range 6-121 months). The high T2 signal in the spinal cord and flow voids decreased or disappeared in 32 (91\%) of 35 patients.

\section{Combined Treatment of edAVFs}

A staged treatment was performed in 3 patients: 1) microsurgical intradural drainer interruption and 2) endovascular embolization of the dilated epidural venous plexus with NBCA. Combined treatment was selected for the 3 patients because of a large dilated epidural venous plexus (2-3 vertebral levels) in 2 patients and multiple bilateral feeders in 1. Postoperative angiography showed complete obliteration of the AVF. No complications occurred in patients receiving the combined treatment. Postoperative MRI showed that the high T2 signal in the spinal cord and flow voids decreased or disappeared in all 3 patients in the median follow-up of 41 months (range 11-51 months).

\section{Neurological Outcomes of edAVFs}

In 81 patients with edAVFs, mRS scores, AminoffLogue gait and micturition grades, and pain/numbness significantly improved, regardless of whether treatment failure occurred, in the median follow-up of 31 months (range 6-121 months) after the index treatment ( $\mathrm{p}<0.001$, Supplemental Table 1). In the 81 patients with edAVFs, an improvement of 1 or more in mRS score or Aminoff- 

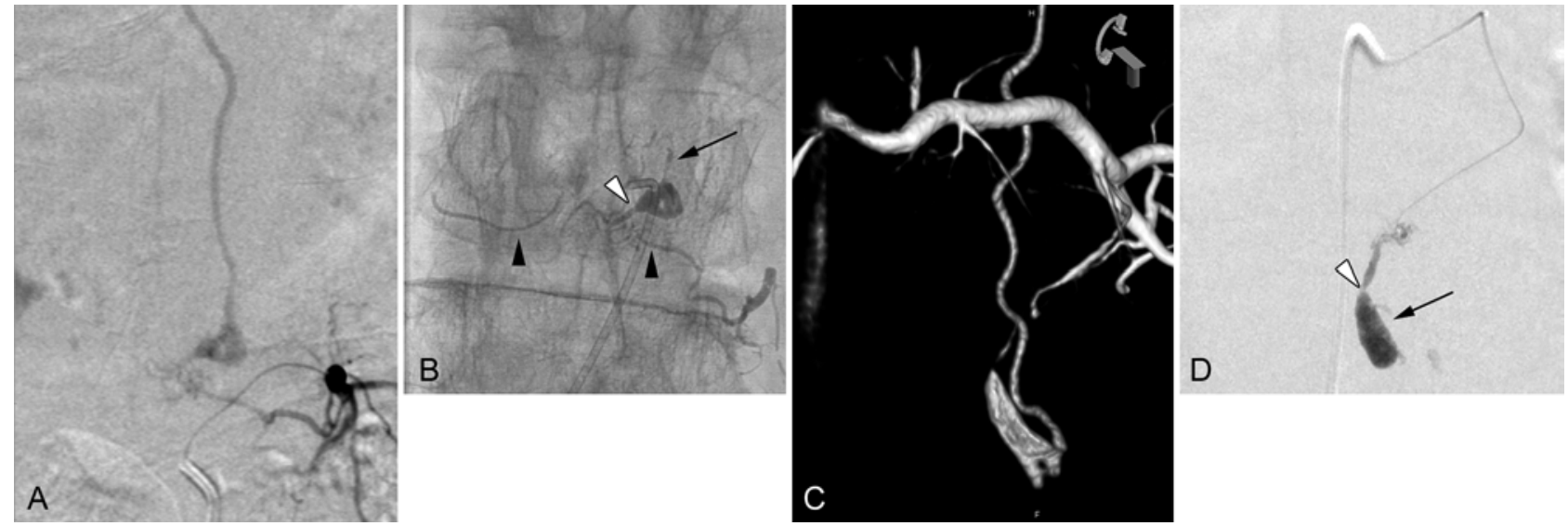

FIG. 6. A and B: Angiograms obtained before (A) and after (B) treatment in an 83-year-old female patient with edAVF. The patient was diagnosed with an edAVF at the L3 level (white arrowhead) fed by bilateral L3 segmental arteries, and she underwent endovascular embolization. The endovascular procedure resulted in the penetration of glue into bilateral feeders (black arrowheads), the dilated epidural venous plexus, and the intradural vein (black arrow), which led to the complete obliteration of the AVF. C and D: Three-dimensional angiogram obtained before treatment $(C)$ and angiogram obtained after treatment (D) in a 41-year-old male patient with an edAVF. The patient was diagnosed with an edAVF at the L3 level (white arrowhead) fed by the left L2 and L3 segmental arteries. He underwent endovascular embolization from the left L2 segmental artery. The endovascular procedure resulted in the penetration of glue into the feeder and the dilated epidural venous plexus only (black arrow). Although glue did not penetrate the intradural vein, this procedure led to the complete obliteration of AVF.

Logue gait or micturition grade was observed in $58(72 \%)$, 59 (73\%), and 47 patients (58\%), respectively.

\section{Discussion}

\section{Major Results}

Based on the largest multicenter cohort, the present study showed key differences in angiographic and clinical characteristics between spinal dAVFs and edAVFs. Spinal epidural AVFs with intradural venous drainage are a distinct entity and may be classified as type V spinal vascular malformations in the context of the original classification system. ${ }^{1,2}$ Furthermore, we herein conducted, for the first time, a direct comparison of the treatment failure rate between microsurgical and endovascular treatment of edAVFs; primary microsurgery was superior to endovascular treatment for initial success.

\section{Key Differences in Angiographic and Clinical Characteristics Between Spinal dAVFs and edAVFs}

Information on spinal edAVFs has been limited to case reports or small case series because of their rarity. ${ }^{6,9,10} \mathrm{Ac}-$ cording to a study of 59 patients by Kiyosue et al., spinal epidural edAVFs often involve the lumbar spine and form a "shunted pouch" in the ventral epidural space that drains into the perimedullary vein, AVFs are predominant in men, and myelopathy is often seen. The present study adds the following important angiographic and clinical results to the existing literature. More than 50\% of edAVFs had multiple feeders, which were associated with treatment failure after the index endovascular treatment. Furthermore, 9\% of edAVFs had multiple intradural venous drainers, which were associated with treatment failure after the index microsurgical treatment. Moreover, preoperative neurological deficits were more severe in patients with edAVFs than in those with dAVFs, although the duration of symptoms did not significantly differ between the 2 groups. Since the spinal level of edAVFs was lower than that of dAVFs, venous reflux into the major lumbosacral radiculomedullary vein may cause more severe venous congestion of the thoracic spinal cord and the conus medullaris. In addition, in patients with edAVFs, the spinal levels of laminectomy, fusion, or fracture that occurred before the diagnosis of edAVFs were adjacent to the fistula level. The development of edAVFs may be associated with a previous history of spinal laminectomy, fusion, or fracture.

\section{Established Treatment Strategy for Spinal dAVFs}

Spinal dAVFs may be treated by microsurgical interruption or endovascular embolization of the intradural venous drainer. ${ }^{11,12}$ Although the success rate of the endovascular treatment of dAVFs has generally increased (46\% in 2004, $72.2 \%$ in 2015 , and $69 \%$ in 2019), meta-analyses have consistently shown that the literature favors microsurgery over endovascular treatment because microsurgery has a higher success rate $(98 \%$ in $2004,96.6 \%$ in 2015 , and $97.2 \%$ in 2019). ${ }^{13-15}$ Regarding spinal dAVFs, microsurgical interruption of the proximal part of the intradural drainer alone is more advantageous for the permanent obliteration of fistulas than endovascular treatment. In microsurgery, even though the fistula on the dura mater is left intact, recurrence has not yet been reported..$^{16}$ In endovascular treatment, the penetration of glue into the intradural proximal drainer is recommended for permanent obliteration of the fistula. ${ }^{17}$

\section{Proposed Treatment Strategy for Spinal edAVFs}

Based on our study results, we propose specific treatment strategies for spinal edAVFs. In patients with a single intradural drainer, microsurgical interruption of the proxi- 
mal part of the intradural vein was sufficient for permanent obliteration of the fistula. In $75 \%$ of patients who underwent drainer interruption, the dilated epidural venous plexus was spontaneously occluded (Fig. 5). Furthermore, in $93 \%$ of patients who underwent microsurgery, the fistula did not recur in the long-term follow-up period (Fig. 4). Therefore, we conclude that, for patients with a single intradural drainer (91\% in our series), microsurgical interruption of the intradural drainer needs to be considered as the first choice of treatment.

In contrast, in endovascular embolization, the penetration of glue into an epidural venous plexus was sufficient for permanent obliteration of the fistula regardless of whether the intradural drainer was occluded (Fig. 6). In edAVFs, the fistulous connection between feeder(s) and the dilated epidural venous plexus was located at a single spinal level in all 81 cases. The reason why the occlusion of the AVF was achieved, even in patients in whom embolic material did not reach the intradural drainer(s), may be explained by the shunt between the feeder(s) and epidural venous plexus being a single AVF itself. Therefore, for patients with multiple intradural drainers $(9 \%$ in our series), endovascular embolization of the enlarged epidural venous plexus needs to be considered as the first choice of treatment.

\section{Neurological Outcomes}

In our cohort, neurological status significantly improved after treatment, regardless of whether initial treatment failure occurred, which perhaps can be explained by the second treatment being performed at a relatively early date (within 5 months) in most patients with initial failure (approximately 80\%). In $7 \%$ of patients treated by microsurgery, postoperative angiography revealed incomplete occlusion because of a new intradural drainer that had not been recognized before the index surgery. In $31 \%$ of patients treated by endovascular embolization, particularly when the index treatment resulted in feeder occlusion only, follow-up angiography revealed incomplete occlusion. Since the diagnostic delay of treatment failure is associated with worse outcomes, follow-up angiography needs to be offered earlier (within 5 months) after the index treatment for all patients with edAVFs.

In patients with edAVFs, postoperative improvements were observed both in gait and micturition disorders, although the severity of the preoperative neurological status was significantly greater in patients with edAVFs than in those with dAVFs. Since spinal edAVFs develop at a lower spinal level than dAVFs, obliteration of venous reflux into the major lumbosacral radiculomedullary vein may improve severe venous congestion of the conus medullaris.

\section{Strengths and Limitations}

The present study has some limitations. Among the 19 centers, there may have been a selection bias in initial treatment selection and variations in the imaging modalities used to detect treatment failure because this was a retrospective analysis of spinal edAVFs between microsurgery and endovascular treatment. Ideally, a prospective randomized study is needed to establish guidelines for the treatment of edAVFs; however, this is difficult due to the rarity of edAVFs. Since our study included the largest number of patients, we consider the results obtained to be of great value for spine surgeons, vascular neurosurgeons, neuroendovascular interventionalists, and neuroradiologists who diagnose spinal edAVFs in clinical practice.

\section{Conclusions}

Although spinal dural and epidural AVFs are similar, there are several important differences that influence the treatment strategy selected. Spinal epidural AVFs with intradural venous drainage are a distinct entity and may be classified as type $\mathrm{V}$ spinal vascular malformations. Based on the largest multicenter cohort of edAVFs, this study showed that primary microsurgery was superior to endovascular treatment for initial treatment success in patients with spinal edAVFs. Microsurgical interruption of the intradural drainer needs to be considered as the first choice of treatment for most patients with a single intradural drainer and endovascular embolization of the enlarged epidural venous plexus as the first choice of treatment for some patients with multiple intradural drainers.

\section{Acknowledgments}

This study was financially supported by the Tokyo Metropolitan Government (grant no. R010603007).

\section{References}

1. Takai K. Spinal arteriovenous shunts: angioarchitecture and historical changes in classification. Neurol Med Chir (Tokyo). 2017;57(7):356-365.

2. Black P. Spinal vascular malformations: an historical perspective. Neurosurg Focus. 2006;21(6):E11.

3. Clarke MJ, Patrick TA, White JB, et al. Spinal extradural arteriovenous malformations with parenchymal drainage: venous drainage variability and implications in clinical manifestations. Neurosurg Focus. 2009;26(1):E5.

4. Nasr DM, Brinjikji W, Clarke MJ, Lanzino G. Clinical presentation and treatment outcomes of spinal epidural arteriovenous fistulas. J Neurosurg Spine. 2017;26(5):613-620.

5. Takai K, Taniguchi M. Comparative analysis of spinal extradural arteriovenous fistulas with or without intradural venous drainage: a systematic literature review. Neurosurg Focus. 2012;32(5):E8.

6. Byun J-S, Tsang ACO, Hilditch CA, et al. Presentation and outcomes of patients with thoracic and lumbosacral spinal epidural arteriovenous fistulas: a systematic review and metaanalysis. J Neurointerv Surg. 2019;11(1):95-98.

7. Kiyosue H, Matsumaru Y, Niimi Y, et al. Angiographic and clinical characteristics of thoracolumbar spinal epidural and dural arteriovenous fistulas. Stroke. 2017;48(12):3215-3222.

8. Aminoff MJ, Logue V. The prognosis of patients with spinal vascular malformations. Brain. 1974;97(1):211-218.

9. Niizuma K, Endo T, Sato K, et al. Surgical treatment of spinal extradural arteriovenous fistula with parenchymal drainage: report on 5 cases. Neurosurgery. 2013;73(2)(Suppl Operative):E287-E294.

10. Takai K, Shojima M, Imai H, et al. Microsurgical and endovascular treatments of spinal extradural arteriovenous fistulas with or without intradural venous drainage. World Neurosurg. 2018;111:e819-e829.

11. Saladino A, Atkinson JLD, Rabinstein AA, et al. Surgical treatment of spinal dural arteriovenous fistulae: a consecutive series of 154 patients. Neurosurgery. 2010;67(5):1350-1358.

12. Sasamori T, Hida K, Yano S, et al. Long-term outcomes after 
surgical and endovascular treatment of spinal dural arteriovenous fistulae. Eur Spine J. 2016;25(3):748-754.

13. Bakker NA, Uyttenboogaart M, Luijckx GJJ, et al. Recurrence rates after surgical or endovascular treatment of spinal dural arteriovenous fistulas: a meta-analysis. Neurosurgery. 2015;77(1):137-144.

14. Goyal A, Cesare J, Lu VM, et al. Outcomes following surgical versus endovascular treatment of spinal dural arteriovenous fistula: a systematic review and meta-analysis. J Neurol Neurosurg Psychiatry. 2019;90(10):1139-1146.

15. Steinmetz MP, Chow MM, Krishnaney AA, et al. Outcome after the treatment of spinal dural arteriovenous fistulae: a contemporary single-institution series and meta-analysis. Neurosurgery. 2004;55(1):77-88.

16. Afshar JK, Doppman JL, Oldfield EH. Surgical interruption of intradural draining vein as curative treatment of spinal dural arteriovenous fistulas. J Neurosurg. 1995;82(2):196-200.

17. Van Dijk JMC, TerBrugge KG, Willinsky RA, et al. Multidisciplinary management of spinal dural arteriovenous fistulas: clinical presentation and long-term follow-up in 49 patients. Stroke. 2002;33(6):1578-1583.

\section{Disclosures}

The authors report no conflict of interest concerning the materials or methods used in this study or the findings specified in this paper.

\section{Author Contributions}

Conception and design: Takai. Acquisition of data: all authors. Analysis and interpretation of data: Takai. Drafting the article: Takai. Critically revising the article: Endo, Yasuhara, Seki,
K Watanabe, Y Tanaka, Kurokawa, Kanaya, Honda, Itabashi, Ishikawa, Murata, T Tanaka, Nishimura, Eguchi, Takami, Y Watanabe, Nishida, Hiramatsu, Ohtonari, Yamaguchi, Mitsuhara, Matsui, Uchikado, Hattori, Horie, Yamahata, Taniguchi.

Reviewed submitted version of manuscript: Endo, Yasuhara, Seki, K Watanabe, Y Tanaka, Kurokawa, Kanaya, Honda, Itabashi, Ishikawa, Murata, T Tanaka, Nishimura, Eguchi, Takami, Y Watanabe, Nishida, Hiramatsu, Ohtonari, Yamaguchi, Mitsuhara, Matsui, Uchikado, Hattori, Horie, Yamahata, Taniguchi. Approved the final version of the manuscript on behalf of all authors: Takai. Statistical analysis: Takai. Study supervision: Takai, Endo, Yasuhara.

\section{Supplemental Information}

Online-Only Content

Supplemental material is available with the online version of the article.

Supplemental Table 1. https://thejns.org/doi/suppl/10.3171/ 2020.2.SPINE191432.

\section{Previous Presentations}

Portions of this work were presented in abstract form at the 34th Annual Meeting of the Neurospinal Society of Japan, Sapporo, Hokkaido, Japan, June 20, 2019.

\section{Correspondence}

Keisuke Takai: Tokyo Metropolitan Neurological Hospital, Tokyo, Japan.takai-nsu@umin.ac.jp. 